\title{
Dystonia and Contractures are Potential Early Signs of CACNA1E-Related Epileptic Encephalopathy
}

\author{
Nelmar V. Ortiz Cabrera ${ }^{a} \quad$ Anna Duat Rodríguez ${ }^{b}$ Bárbara Fernández Garoz \\ Beatriz Bernardino Cuesta $^{\mathrm{b}}$ María Jiménez Legido ${ }^{\mathrm{b}}$ Verónica Cantarín \\ Extremera $^{\text {b Juan J. García Peñas }}{ }^{\text {b }}$
}

${ }^{a}$ Clinical Genetics, Hospital Infantil Universitario Niño Jesús, Madrid, Spain; ${ }^{b}$ Neurology Department, Hospital Infantil Universitario Niño Jesús, Madrid, Spain; ${ }^{\circ}$ Department of Laboratory Medicine, Hospital Infantil Universitario Niño Jesús, Madrid, Spain

\section{Established Facts}

- Pathogenic variants in CACNA1E are related to early infantile epileptic encephalopathy with macrocephaly, hypotonia, and hyperkinetic movements.

- Most of the variants described in CACNA1E are gain of function variants, and the role of haploinsufficiency has yet to be clarified.

\section{Novel Insights}

- Early-onset dystonia with global developmental delay and areflexia may precede the appearance of epileptic encephalopathy.

- A genotype-phenotype correlation could exist for different locations of pathogenic missense variants in this gene.

\section{Keywords}

CACNA1E - Exome sequencing · Intellectual disability · Early onset epileptic encephalopathy

\section{Abstract \\ Epileptic encephalopathy related to CACNA1E has been de- scribed as a severe neurodevelopmental disorder present- ing with early-onset refractory seizures, hypotonia, macro- cephaly, hyperkinetic movements, and contractures and is}

associated with an autosomal dominant inheritance pattern. Most pathogenic variants described to date are missense variants with a gain of function effect, and the role of haploinsufficiency has yet to be clarified. We describe 2 cases of CACNA1E encephalopathy. Notable findings include congenital contractures and movement disorders predating onset of epilepsy, particularly dystonia. We further compared the key phenotypic features depending on variant location. In conclusion, the appearance of congenital contractures, areflexia, and movement disorders before the onset of epi-
Nelmar Valentina Ortiz Cabrera

Clinical Genetics Hospital Infantil Universitario Niño Jesús 
lepsy may provide key guidance in the diagnosis of epileptic CACNA1E encephalopathy. A genotype-phenotype correlation was found between the presence of movement disorders and severe intellectual disability and the location of the variant in the CACNA1E gene.

(c) 2020 S. Karger AG, Basel

\section{Introduction}

In 2018, Helbig et al. [2018] described epileptic encephalopathy related to CACNA1E (OMIM \#618285), studying 30 patients with 14 de novo pathogenic variants in the CACNA1E gene (MIM*601013; 1q25). In this channelopathy, heterozygous variants in the CACNA1E gene cause a severe neurodevelopmental disorder presenting as early-onset refractory seizures, hypotonia, macrocephaly, hyperkinetic movements, and contractures. CACNA1E is inherited according to an autosomal dominant pattern, although, due to the severity of the phenotype, all patients described have a de novo variant [Helbig et al., 2018]. The vast majority of pathogenic variants are missense variants with an apparent gain of function effect and cluster within the cytoplasmic ends of all 4 S6 segments [Carvill, 2019], with the recurrent variant c.1054G >A; p.Gly352Arg found to be present in 9 out of $30(60 \%)$ patients in the study [Helbig et al., 2018]. The role of haploinsufficiency has not been clarified to date.

This paper describes 2 unrelated patients with early infantile epileptic encephalopathy due to CACNA1E, comparing their genotype and phenotypic to all cases published up to July 2020 in order to determine a possible phenotype-genotype correlation.

\section{Case Presentation}

Case 1. A 2-year-4-month-old male born to healthy and nonconsanguineous parents with no relevant family history presented with congenital talipes equinovarus and subtle shortening of the long bones. At 2 weeks of age, he began to experience episodes of dystonia several times a day with a tendency toward opisthotonos, spinal hyperextension, internal rotation of the upper limbs, and irritability. He presented global developmental delay, poor eye contact, noncongenital macrocephaly (head circumference at birth $36.6 \mathrm{~cm}+1 \mathrm{SD}$; head circumference at 2 months $42 \mathrm{~cm}+2.7 \mathrm{SD}$ ), and severe axial hypotonia (Fig. 1). At 6 months of age, the patient developed epileptic spasms with hypsarrhythmia. His symptoms evolved into dystonic cerebral palsy with areflexia and a profound developmental delay with no motor or social milestones achieved at 2 years of age, and he required a gastrostomy due to a feeding disorder. His epilepsy was refractory to multiple antiepileptic drugs (i.e., vigabatrin, corticosteroids, valproic acid, zonisamide, topiramate), and he also received pyridoxine and was initiated on a ketogenic diet (KD). Manifestations consisted of spasms and tonic seizures. Facial features included a flattened philtrum, frontal bossing, features consistent with hypotonic facies, epicanthus, and preauricular pits (Fig. 1a). Dystonia (twisting and repetitive movements) progressed, and the patient presented with choreoathetoid movements that were more noticeable with voluntary movement. The frequency and intensity of his dystonia symptoms and irritability have decreased over time.

Case 2. The patient is a 22-month-old female born to healthy and nonconsanguineous parents with no relevant family history. Prenatal development was uneventful except for talipes equinovarus. At 1 month of age, she presented with dystonic episodes with hyperextension of the trunk, internal rotation of the upper extremities, and progressive irritability. She also presented global developmental delay, poor eye contact, severe axial hypotonia, and oculogyric spasms not correlated with electroencephalographic recordings. At 4 months of age, the girl developed epileptic spasms and hypsarrhythmia. Her symptoms also evolved into dystonic cerebral palsy with areflexia, profound developmental delay (no motor or social milestones achieved), and epilepsy that was refractory to multiple antiepileptic drugs (valproic acid (VPA), levetiracetam, clobazam, vigabatrin, zonisamide, lamotrigine, and topiramate), and she also received pyridoxine and $\mathrm{KD}$; her seizure types included spasms and tonic seizures. Other features were frontal bossing, hypotonic facies, microretrognathia, puffy cheeks, a flattened philtrum, and dolichocephaly without macrocephaly. The patient also required a gastrostomy due to a feeding disorder (Fig. 1b). Lastly, she also sustained an unexplained or stress fracture in the left femur and was diagnosed with osteopenia.

Laboratory tests of blood, urine, and spinal fluid were performed in both patients to study a) intermediate metabolism, e.g., amino acid disorders, organic acid disorders, carbohydrate metabolism, creatine, purines and pyrimidines, and neurotransmitters; $b$ ) defects of $B$-oxidation of fatty acids; c) congenital disorders of glycosylation (CDG); and d) peroxisomal diseases. All results were normal. Karyotype and CGH array $180 \mathrm{~Kb}$ were normal. Cerebral and spinal magnetic resonance imaging (MRI) scans in both patients were normal at 2 months and when
Ortiz Cabrera et al. 

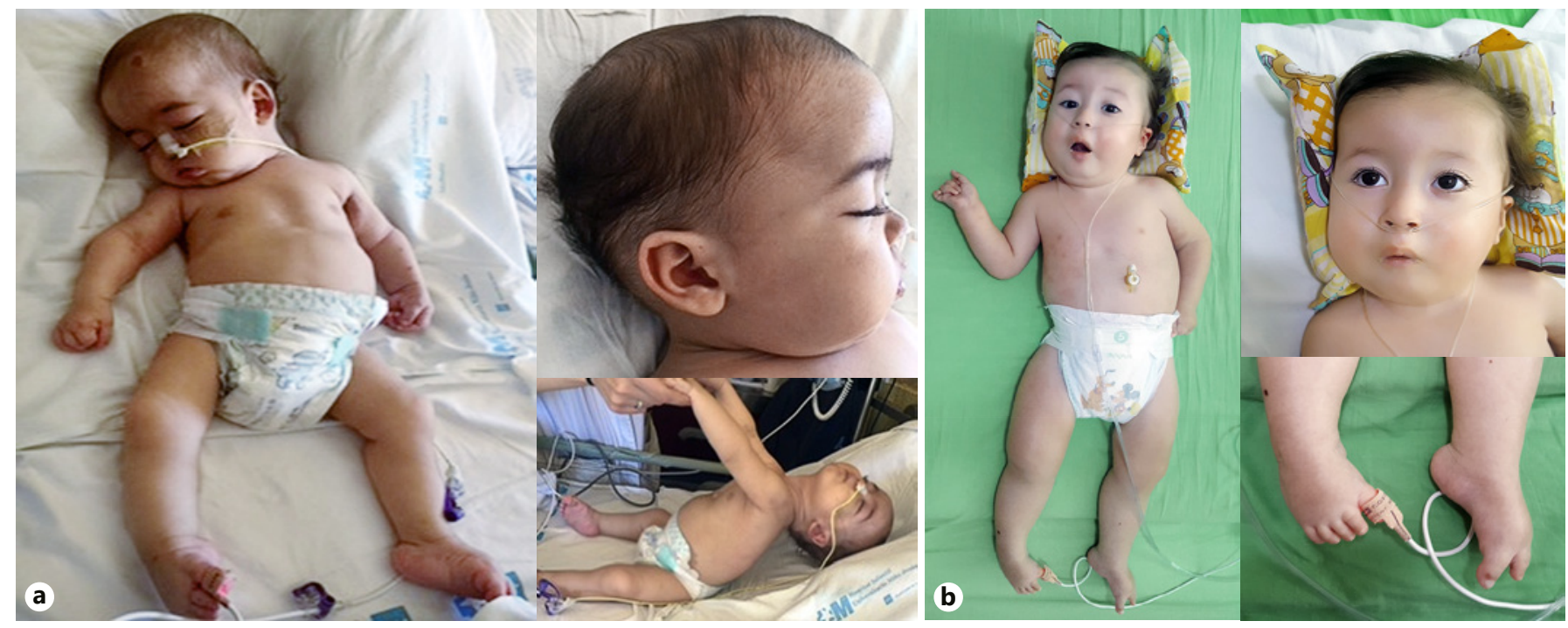

Fig. 1. a. Phenotype of case 1 showing severe axial hypotonia, frontal bossing, low set ears, long and smooth philtrum, subtle rhizomelic shortening of limbs, and congenital talipes equinovarus. b. Phenotype of case 2 revealing microretrognathia, low set ears, frontal bossing, congenital talipes equinovarus, and severe hypotonia.

repeated at 6 months in case 1 and at 4 months in case 2. Eye fundus and visual and auditory evoked potentials were normal in both patients. Electromyogram and electroneurogram findings were also normal in both patients.

\section{Results}

In both patients, diagnostic singleton whole-exome sequencing (WES) was performed in 2 different commercial laboratories (CENTOGENE, Germany and IMEGEN, Spain), though the primary analysis was conducted on a list of genes related to CDG in case 1 and epileptic encephalopathies in case 2 . No pathogenic variants were reported in either case. We subsequently performed an unbiased analysis of the WES data for both patients using phenotypes in accordance with the following Human Phenotype Ontology (HPO) terms: dystonia HP:0001332, global developmental delay HP:0001263, seizure HP:0001250, and talipes equinovarus HP:0001762. We observed the heterozygous missense pathogenic variant c.1054G >A; p.Gly352Arg in the CACNA1E gene in both patients. In case 1 the variant had a mutant allele fraction of $55 \%$ (22/40 reads) and in case 2 the variant had a mutant allele fraction of 45\% (23/51 reads). Using Sanger sequencing, parental DNA was studied, revealing a de novo variant in CACNA1E in both patients.
We compared the clinical features described for the group studied by Heldig et al. [2018] with those of our 2 patients (Table 1) and attempted to identify potential genotype-phenotype correlations (Table 2). Using Fisher's exact test and a statistical significance level of 0.05 , we compared every phenotypic feature for a specific domain against all other locations. A movement disorder was present in $43 \%$ of all patients and in all subjects with variants located at the cytoplasmatic topological domain between DI and DII. This location included the p. Gly352Arg variant, and movement disorders in this group of patients were statistically more frequent $(p<$ 0.05 ) than in patients with variants in other protein locations. To the contrary, the presence of movement disorders were statistically less frequent $(p<0.05)$ in patients with variants located at DII, including the recurrent variant p.Ala702Thr located at the S6 transmembrane domain of DII.

Also, the presence of severe intellectual disability was significantly less common in the group of patients with variants located in the cytoplasmic topological domain between DI-II and DIV than in individuals with variants located elsewhere in the protein (50 vs. $93 \%$; Table 2).

Refractory epilepsy, contractures, and macrocephaly was present in 90,52 , and $40 \%$, respectively. No differences in the frequency of these features were detected. 


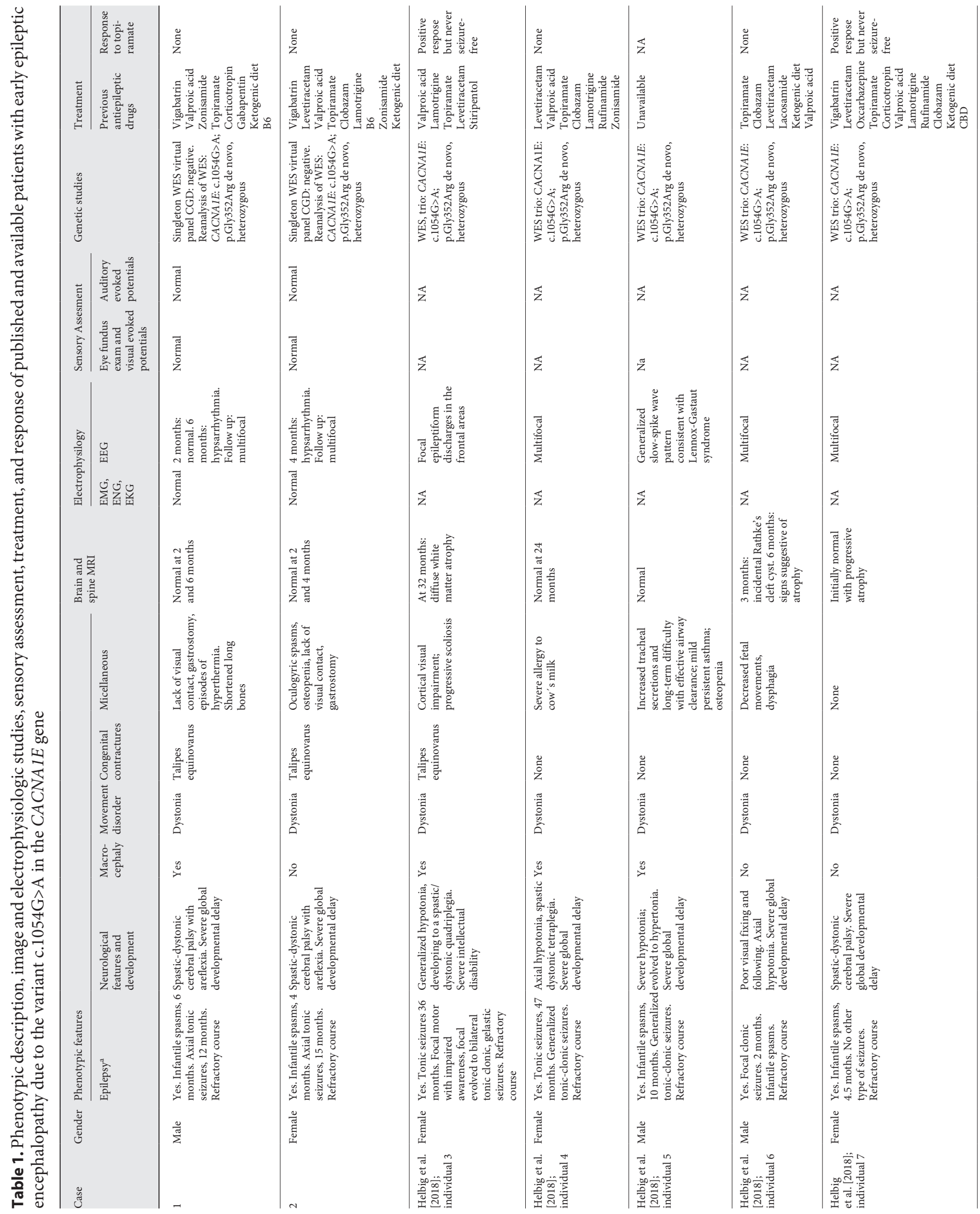




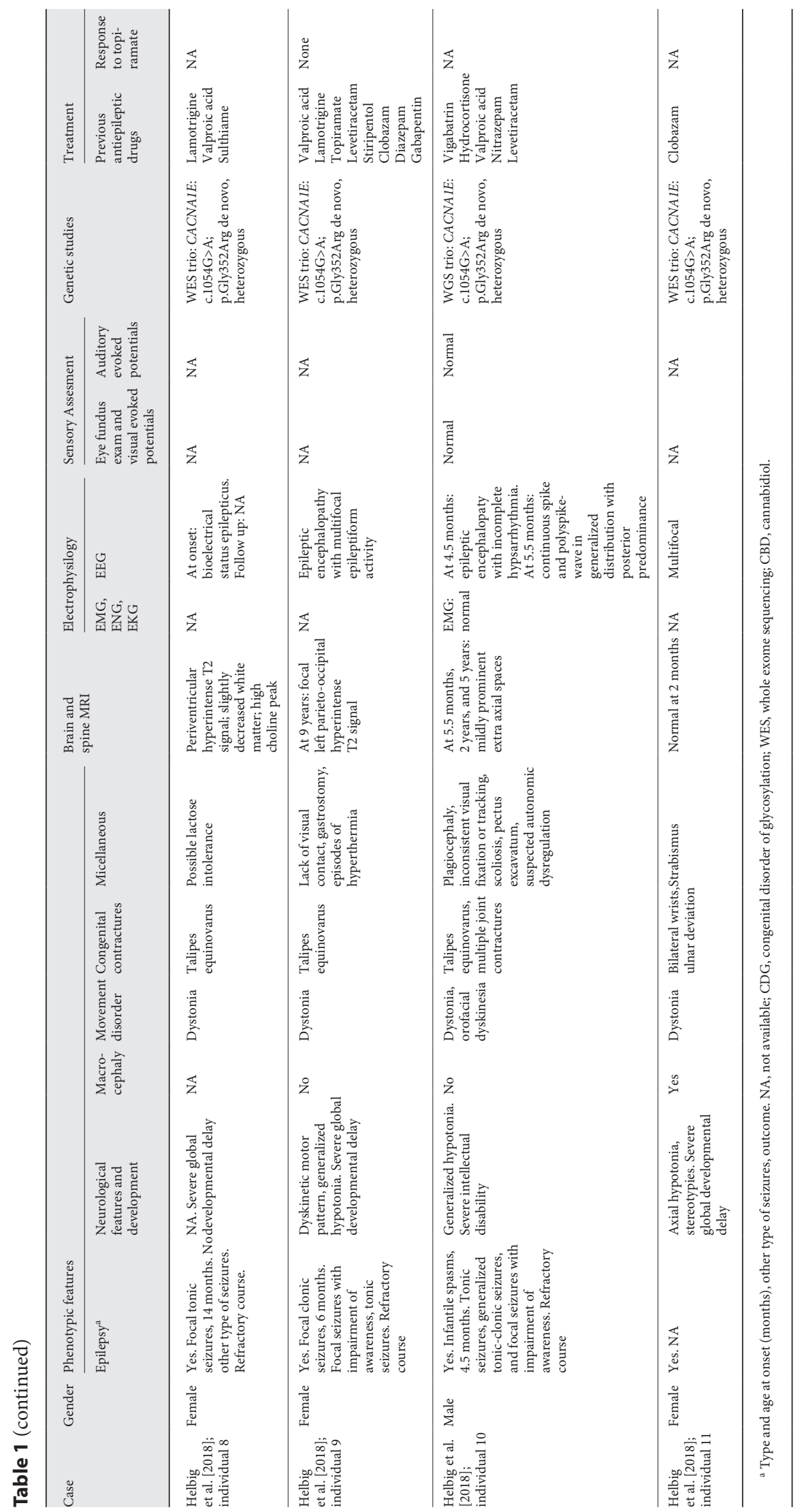




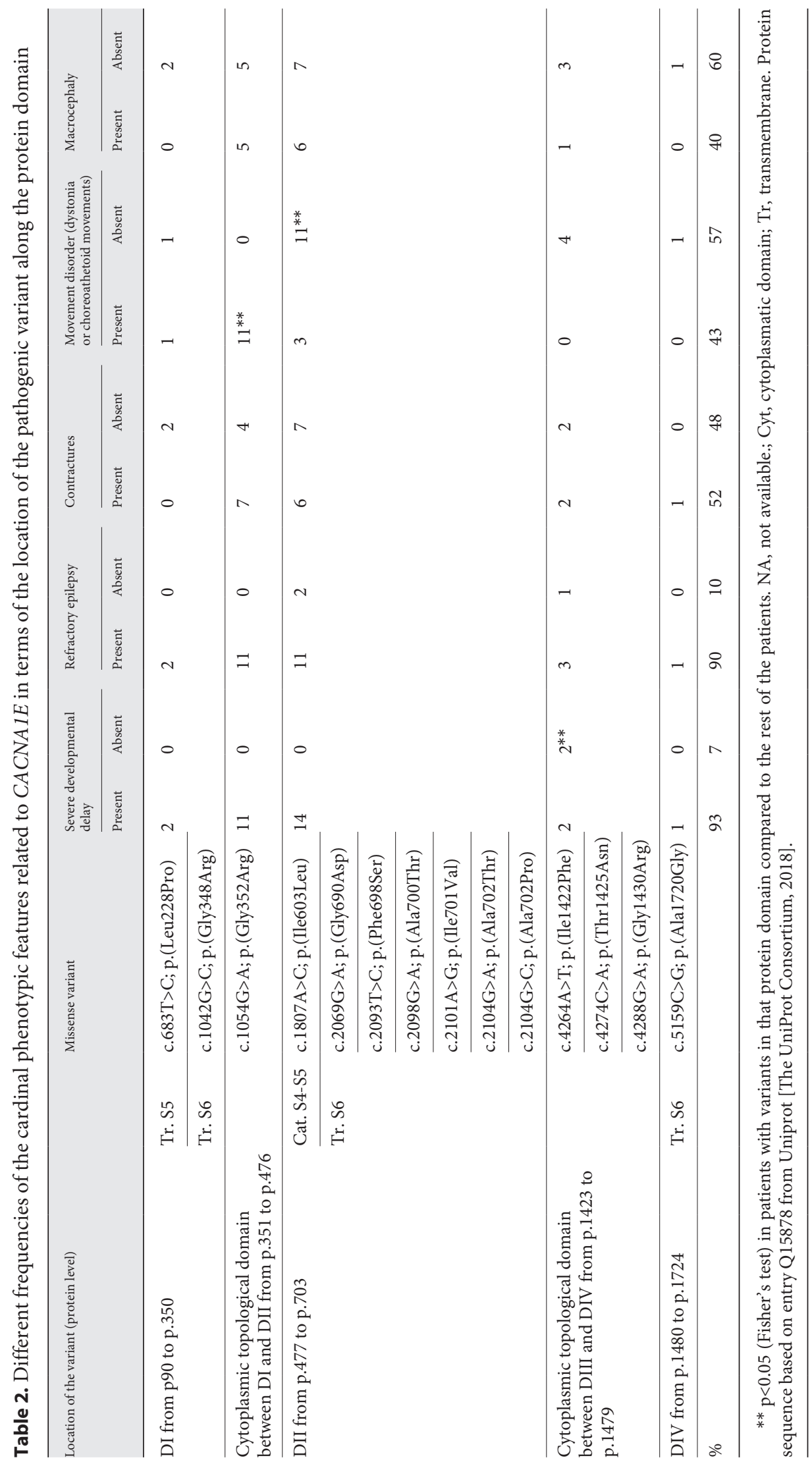




\section{Discussion}

Early-onset epileptic encephalopathy (EOEE) represents a heterogeneous group of disorders in which epilepsy appears in the first 6 months of life and is usually associated with a severe neurodevelopmental disorder, among other symptoms [Heyne et al., 2018; Carvill, 2019]. Currently, a genetic etiology can be identified in about $30 \%$ of cases [Mercimek-Mahmutoglu et al., 2015; Balciuniene et al., 2019], mainly as pathogenic variants in genes encoding neuronal ion channels, proteins involved in synaptic transmission, or transcriptional regulatory proteins [Mercimek-Mahmutoglu et al., 2015; Heyne et al., 2018; Balciuniene et al., 2019].

Calcium channels are critical for neuronal function and different types of channels have different functions. High voltage-activated (HVA) channels open in response to large membrane depolarizations [Simms and Zamponi, 2014]. HVA channels have a complex, tissue-specific structure where the main components are a) a subunits, the primary pore-forming subunits of the calcium channel; b) $\beta$ subunits linked to the $\alpha$ subunit in the intracytoplasmic environment regulating channel opening; c) the $\alpha 2 \delta$ subunit attached to the extracellular leaflet of the plasma membrane via a glycophosphatidylinositol (GPI), which augments channel cell surface density; and d) the $\gamma$ subunit, which includes transmembrane domains, the function of which is less clear [Simms and Zamponi, 2014; Wu et al., 2015].

The a subunits are encoded by the following 10 genes: CACNA1A, CACNA1B, CACNA1C, CACNA1D, CACNA1E, CACNA1F, CACNA1G, CACNA1H, CACNA1I, and CACNA1S [Carvill, 2019]. These subunits are made up of 4 homologous domains with 6 transmembrane domains each, where the transmembrane segments 5 (S5) and 6 (S6) line the inner pore of the channel [Carvill, 2019]. Each gene has a cell- and tissue-specific expression pattern. CACNA1E is highly expressed in the central nervous system and encodes the $\alpha 1$-subunit of the HVA, rapidly inactivating the R-type calcium channel that initiates synaptic transmission [Helbig et al., 2018].

The pathogenic variant in CACNA1E found in our patients, c.1054G>A; p.(Gly352Arg), was first described by Helbig et al. [2018] in 9 patients (Table 1). Raybaud et al. [2006] demonstrated that this variant, which is located at the cytoplasmic topological domain between DI and DII, causes a gain of function that favors voltage-dependent activation and diminishes the rate of voltage-dependent inactivation. The role of haploinsufficiency has yet to be clarified.

Early and Relevant Clinical Data on

CACNA1E Epileptic Encephalopathy
Some aspects of CACNA1E encephalopathy are phenotypically similar to other early epileptic encephalopathies, including early-onset refractory epileptic seizures (primarily spasms and axial tonic seizures), in whom the disease tends to be refractory and presents with severe developmental delay and abnormalities in muscle tone. However, $C A C N A 1 E$ encephalopathy has unique features:

a) Dystonia is a common symptom in patients with CACNA1E encephalopathy, especially those carrying the c.1054G >A; p.(Gly352Arg) recurrent variant, as in the case of both patients described in this paper and all patients in Helbig's group [Helbig et al., 2018]; b) Congenital contractures are present in 52\% (16/31) of patients with CACNA1E (Table 2); c)Macrocephaly was evident in $40 \%(12 / 30)$ of patients (Table 2); d) Deep tendon areflexia, as presented by both patients described here, with normal peripheral electroneurogram.

In the 9 patients with the c.1054G >A; p.(Gly352Arg) variant described by Helbig et al. [2018], no deep tendon reflexes are mentioned. However, 1 patient with the variant c.683T $>$ C; p.(Leu228Pro) had brisk reflexes and 1 with the variant c.1807A $>$ C; p.(Ile603Leu) presented hyporeflexia, while the rest of the patients were described as having normal reflexes or no information of reflexes was provided.

Although we describe only 2 patients, both were phenotypically very similar and presented with the movement disorder and contractures at early stages. We believe these 2 features could aid in identifying this group of patients early in the course of the disease.

In case 2, the cause of osteopenia has not been elucidated, though its relation to CACNA1E seems unlikely and could be explained by a multifactorial complication of chronic immobility, the effect of drugs, and $\mathrm{KD}$ [Bachrach and Gordon, 2016].

\section{Conclusion}

Epileptic encephalopathies continue to pose a significant challenge for etiology and diagnosis, and a direct genetic approach is difficult. It is of great interest to identify early and specific phenotypic data in those entities that could be relevant. Congenital contractures and presence of a movement disorder prior to the onset of epilepsy may be the key to guiding the diagnosis of epileptic CACNA1E encephalopathy. In this study, we found a genotype-phenotype correlation between movement disorders and the presence of severe intellectual disability with a variant in the CACNA1E gene. 


\section{Acknowledgements}

We thank the children and their families who participated in the study.

We thank the Biomedical Research Foundation Niño Jesús and $\mathrm{O}$. Shaw for the assistance in reviewing and editing the present manuscript.

\section{Statement of Ethics}

This work was conducted ethically in accordance with the World Medical Association Declaration of Helsinki. All patients (or their parents or guardians) have given written informed consent for the use of patient clinical data and images.

\section{Conflict of Interest Statement}

The authors have no conflicts of interest to declare.

\section{Funding Sources}

Whole exome sequencing performed on the patients' DNA samples and all other analyses were funded by non-discretionary grants.

\section{Author Contributions}

All authors have substantially contributed to the study. N.V.O.C., A.D.R., and B.F.G. designed the study, performed the literature search, and drafted the initial manuscript. B.B.C., V.C.E., and M.J.L. participated in patient recruitment and gathering of clinical data. J.J.G.P., N.V.O.C., and A.D.R. conceptualized the study and reviewed the manuscript.

\section{References}

Bachrach LK, Gordon CM. Section on Endocrinology. Bone Densitometry in Children and Adolescents. Pediatrics. 2016; 138(4): e20162398.

Balciuniene J, DeChene ET, Akgumus G, Romasko E, Cao K, Dubbs H, et al. Use of a dynamic genetic testing approach for childhood-onset epilepsy. JAMA Netw Open. 2019;2(4): e192129.

Carvill GL. Calcium channel dysfunction in epilepsy: Gain of CACNA1E. Epilepsy Curr. 2019;19(3):199-201.
Helbig KL, Lauerer RJ, Bahr JC, Souza IA, Myers CT, Uysal B, et al. De novo pathogenic variants in CACNA1E cause developmental and epileptic encephalopathy with contractures, macrocephaly, and dyskinesias. Am J Hum Genet. 2018;103(5):666-78.

Heyne H, Singh T, Stamberger H, Abou Jamra R, Caglayan H, Craiu D, et al. De novo variants in neurodevelopmental disorders with epilepsy. Nat Genet. 2018;50(7):1048-53.

Mercimek-Mahmutoglu S, Patel J, Cordeiro D, Hewson S, Callen D, Donner EJ, et al. Diagnostic yield of genetic testing in epileptic encephalopathy in childhood. Epilepsia. 2015; 56(5):707-16.
Raybaud A, Dodier Y, Bissonnette P, Simoes M, Bichet DG, Sauve $R$, et al. The role of the GX9GX3G motif in the gating of high voltage-activated $\mathrm{Ca} 2+$ channels. J Biol Chem. 2006;281(51):39424.

Simms BA, Zamponi GW. Neuronal voltage-gated calcium channels: structure, function, and dysfunction. Neuron. 2014;82(1):24-45.

The UniProt Consortium. UniProt: A worldwide hub of protein knowledge. Nucleic Acids Research. 2018;47(D1):D506-D515.

Wu J, Yan Z, Li Z, Yan C, Lu S, Dong M, et al. Structure of the voltage-gated calcium channel Cav1.1 complex. Science. 2015;350(6267): $\operatorname{aad} 2395$. 